Die Moderne und Platon 
Arbogast Schmitt

\section{Die Moderne und Platon}

Verlag J. B. Metzler

Stuttgart - Weimar 
Bibliografische Information Der Deutschen Bibliothek Die Deutsche Bibliothek verzeichnet diese Publikation in der Deutschen Nationalbibliografie; detaillierte bibliografische Daten sind im Internet über $<\mathrm{http} / / \mathrm{ddb} . \mathrm{de}>$ abrufbar.

ISBN 978-3-476-01949-3

ISBN 978-3-476-02926-3 (eBook)

DOI 10.1007/978-3-476-02926-3

Dieses Werk einschließlich aller seiner Teile ist urheberrechtlich geschützt. Jede Verwertung außerhalb der engen Grenzen des Urheberrechtsgesetzes ist ohne Zustimmung des Verlages unzulässig und strafbar. Das gilt insbesondere für Vervielfältigungen, Übersetzungen, Mikroverfilmungen und die Einspeicherung und Verarbeitung in elektronischen Systemen.

(C) 2003 Springer-Verlag GmbH Deutschland Ursprünglich erschienen bei J. B. Metzlersche Verlagsbuchhandlung und Carl Ernst Poeschel Verlag GmbH in Stuttgart 2003

www.metzlerverlag.de info@metzlerverlag 
"Obwohl die Vernunft für alle ein und dieselbe ist, leben die meisten, als hätte jeder seine Privatvernunft." Heraklit

„Man versteht Bücher besser und macht weniger Fehler, wenn man beim Unterscheiden auf das Gemeinsame achtet." Nikolaus von Kues 


\section{Inhaltsverzeichnis}

Vorwort

$\begin{array}{ll}\text { Zur Einführung } & 7\end{array}$

1 Schwierigkeiten in der Begriffsbestimmung und im Selbstverständnis der ,Moderne' - und die Entstehung des ,geschichtlichen Denkens'

2 Die Wende zum Diesseits und die Erhöhung der Natur zur ,schönen' Natur in der Kunst der Neuzeit

3 Die Wende zur Erfahrung und die Erhöhung des Einzeldings zum ,wohlbestimmten' Ding in den wissenschaftlichen Diskursen der ,Moderne'

4 Die Wende zur Erfahrung und die Entstehung des ,modernen' Begriffs des Denkens: das Bewußtsein Bewutßtseins- und Unterscheidungsphilosophien. Über eine Grunddifferenz möglicher Erkenntnisbegründungen in der antiken und modernen Philosophie

6 Bestimmtheit und Unterscheidbarkeit als philosophisches Grundprinzip bei Platon und Aristoteles

7 Die Renaissance: nicht die Wiedergeburt ,der' Antike, sondern die Wiederbelebung der hellenistischen Antike 66

8 Zum Aufbau dieses Buches: Teil I 70

9 Zum Aufbau dieses Buches: Teil II 74

I. TEIL "Abstraktes Denken - Konkrete Sinnlichkeit": Zum Gegensatz von Kultur und Natur in der Moderne

I Machen Freiheit und Unbestimmtheit den Menschen zu einem Kulturwesen? oder: Warum die Antike antiquiert erscheint

1 Der Gegensatz von selbstgeschaffener Kultur und determinierter Natur im Menschen

$\begin{array}{ll}1 \text { a. bei Vico } & 81\end{array}$

1 b. bei Herder 83

1 c. bei Cassirer und den gegenwärtigen Kulturwissenschaften $\quad 88$

$1 \mathrm{~d}$. Brüche und Gemeinsamkeiten in den neuzeitlich-modernen Diskursen über den Menschen als Kulturwesen

1 e. Wirkungsgeschichtlich begründete Zweideutigkeiten in der Bestimmung des Kulturwesens ,Mensch'

1f. Der ,neue' Freiheitsbegriff: Resultat der Verabsolutierung der geschichtlichen Welt 
1 g. Die Begründung des Gegensatzes von Natur und Kultur in der Entgegensetzung der rezeptiven und spontanen Vermögen des Menschen

1 h. Die ,Entdeckung' der Spontaneität des reflexiven Denkens und der Bruch mit der Geschichte

2 Die Verengung des Begriffs der Rationalität durch den Gegensatz von ,Sinnlichkeit' und ,Verstand'

2a. Die ,neue' Rationalität als Abgrenzungskriterium gegen ,die' Antike

2 b. Der ,Begriff' als Ordnungskriterium gegenständlicher Anschauungen - zum Ansatz einer Kritik am neuzeitlichen Rationalitätsverständnis

2c. Die Wende zur Praxis

II Der ,gesunde Menschenverstand' und die NaturKultur-Antithese

1 Zum Ansatz einer Kritik an der neuzeitlichen Natur-KulturAntithese

Über die Urschuld der Rationalität

3 Das Neue der Neuzeit: Befreiung von der Anschauung oder von der Herrschaft des Begriffs?

4 Aporien im Verhältnis von Anschauung und Denken - in moderner und antiker Perspektive

4a. Zur Sinneserfahrung

4 b. Zur Vorstellung als ,Verstand'

5 Probleme der Begriffsbildung: Konstellationen und Lösungsansätze

6 Der Vorrang der Sinneserkenntnis vor dem Denken 142

6a. in der Erkenntnistheorie des antiken Empirismus - am Beispiel von Lukrez, De rerum natura

6 b. in der ,ästhetischen Moderne' - am Beispiel Baumgartens 143

6 c. in der Kunsttheorie des Naturalismus 145

6 d. im logischen Empirismus der, Wiener Schule' - am Beispiel Rudolf Carnaps

6 e. Die Bedeutung der Zeit für die Begriffsbildung in der Philosophie seit der Aufklärung 166

7 Zusammenfassende Beurteilung und Überleitung 178

7 a. Die Erfahrung von Einzeldingen als Bezugspunkt aller Erkenntnis bei Kant und der Anfang des Erkennens bei Aristoteles

7 b. Die Erhebung von bewiesenen Gegenständen (Ich, Raum, Zeit usw.) zu Beweiskriterien

7 c. Die Erhebung des Bewußtseins zum Inbegriff der Rationalität des Menschen: Die Folge einer Verwechslung einer Wirkung des Denkens mit dem Denken selbst 
II. TEIL „Konkretes Denken“ als Voraussetzung einer Kultur der Ethik, der Politik und der Ökonomie bei Platon und Aristoteles

I Zur Deutung der ,Antike' aus der Perspektive neuzeitlicher Rationalität

II Die erkenntnistheoretischen Grundlagen einer Unterscheidungsphilosophie

1 Das Widerspruchsaxiom als Grundkriterium der Rationalität bei Aristoteles

2 Rationales Denken und geschichtliches Verstehen bei Platon

4 Das Unterscheiden als Grundakt des Denkens und das Wissenschaftssystem der ,Freien Künste'

4 a. Wahrheit ist Übereinstimmung von Denken und Gegenstand' - in platonischer und nachcartesianischer Deutung

$4 \mathrm{~b}$. Die methodische Bedeutung des Widerspruchsaxioms und die ,nichtklassischen' Logiken der Gegenwart

III Abstraktes Bewußtsein - Konkretes Denken: Zur Überwindung des Gegensatzes von Gefühl und Verstand in einer Unterscheidungsphilosophie

1 Zur Erweiterung des Begriffs des Denkens

2 Die eigentümliche Leistung des Denkens. Ihre Unabhängigkeit vom Gegensatz des Bewußten und Unbewußten

IV Die Seele in bewußtseinsphilosophischer und unterscheidungsphilosophischer Analyse

1 Verstand, Gefühl und Wille und ihr Zusammenwirken beim Handeln

2 Die Intelligenz von Emotionen und Motivationen in der Moderne und in der platonisch-aristotelischen Psychologie

V Die verschiedenen Formen des Wollens und ihre Abhängigkeit vom Erkennen

1 Der unabhängige, ,freie' Wille in der Stoa

2 Begehren, Sich-Ereifern und vernünftiges Wollen als Grundmöglichkeiten psychischen Verhaltens bei Platon

3 Die Erkenntnisbedingungen des Begehrens und SichEreiferns: Wahrnehmung, Gegenstandsanschauung und Meinung 
3a. Die eigentümliche Leistung der Wahrnehmung als Wahrnehmung

3 b. Die ,Wahrnehmung' von Gegenständen, Situationen und ,Gefühlen'

3 c. Die erkenntnistheoretische Analyse des Meinens (doxa, opinio) durch Platon und Aristoteles

3 d. Zur Differenz der Gegenstände von Wahrnehmung und Meinung

3 e. Zum Unterschied von ,Anschauung' und Meinung

4 Meinung und Emotionalität

VI Die ästhetische, ethische und politische Bedeutung einer Kultur der Gefühle bei Platon und Aristoteles 341

1 Reduziert Aristoteles Gefühle auf abstrakte Lusterfahrungen?

Die Entstehung und das Bewußtsein von Gefühlen

3 Abstrakte Gefühle - mit Beispielen aus Euripides und Sophokles

4 Rationale Gefühle. Ein Beispiel: Aristoteles' Analyse der Selbstliebe

Kunst als Erziehung der Gefühle - das Beispiel der Tragödie 361

- Die Furcht $\quad 365$

- Das Mitleid 369

- Die staatspolitische Bedeutung von Mitleid und Furcht

VII Theorie und Praxis: Zur Grundlegung der Staatsund Gesellschaftstheorie in einer Theorie des Menschen durch Platon und Aristoteles

1 Der Staat als Bedingung der Möglichkeit der Verwirklichung von Freiheit und Individualität

2 Der Vorrang des Individuums vor der Gemeinschaft in der von Homer geschilderten Gesellschaft

2 a. Konventionelle oder postkonventionelle Ethik bei Homer? 385

2b. Die Schädigung der Gemeinschaft: Eine Folge der Verfehlung des privaten Glücks

2c. Die homerische Psychologie und die Unterscheidung von wahren und scheinbaren Vorteilen

3 Der Vorrang des Individuums vor dem Staatsganzen bei Platon

4 Individualität als gegebenes Faktum oder als Aufgabe 403

4 a. Kriterien der Ermittlung des sog. ,primären' und ,immanenten' Allgemeinen 407

$4 \mathrm{~b}$ Das wissenschaftliche Allgemeine bei Aristoteles und die platonische Idee 
$4 \mathrm{c}$ Das wissenschaftliche Allgemeine und die Bestimmung von Individualität

5 Individuelles Glück und Gerechtigkeit im Staat

6 Selbsterhaltung und individuelles Glück: zum Unterschied zwischen Überleben und gutem Leben - Einführung in einen Grundunterschied der Staats- und Wirtschaftstheorie bei Platon und Aristoteles und in der Neuzeit

6 a. "Das Interesse bestimmt die Theorie": Das stoischneostoische Willenskonzept und seine Bedeutung für die Wirtschaftstheorie bei Adam Smith und die Staatstheorie bei Thomas Hobbes

Die Sympathie aller mit allen als Korrektiv gegen die Egoismen des ,Marktes'? - Zur Verbindung von Ökonomie und Ethik in der liberalistischen Markttheorie bei Adam Smith

6 b. Das bonum privatum als axiomatische Grundlage ethischen und ökonomischen Handelns bei Aristoteles

c. Die neuzeitliche, Wende' zur Wohlfahrt des Einzelindividuums und ihre Aporien

- Hobbes' ,neue' Theorie und ihr Verhältnis zur Tradition

- Die Reduktion des Menschen auf den Selbsterhaltungstrieb und die Verwissenschaftlichung dieses Menschenbildes

VIII Evolutionsbiologische Bedingungen der Selbsterhaltung und rationale Bedingungen der Selbstverwirklichung des Menschen - Plädoyer für eine Neubewertung der Rationalität

1 Zur Uminterpretation von Zufall, Scheitern, Bosheit und Vernichtung des Einzelnen in einen Dienst für die Entwicklung des Ganzen

1 a. Über das Gemeinsame im stoischen Dogmatismus und in der Evolutionstheorie bei der Beurteilung des Verhältnisses von Zufall und Notwendigkeit

1 b. Würden Teufel, um zu überleben, eine Republik gründen? Zum Ansatz einer Differenzierung zwischen natürlichem Selbsterhaltungstrieb und einem rational begründeten Streben nach Selbsterhaltung

2 Naturgeschichte und Geschichte bei Aristoteles

2a. Die Evolution und ihre rationalen Vorgaben bei der Entstehung und Entwicklung, natürlicher' Dinge

2 b. Sind Bio-Elemente, platonische Formen'?

2c. Rationale Bedingungen für die Genese und Ausbildung menschlicher Vermögen

3 Rationale Selbstverwirklichung als Bedingung des Zusammenfallens des privaten Vorteils mit dem Wohl des Ganzen 
4 Wunsch und Bedarf in der Wirtschafts- und Gesellschaftstheorie des Aristoteles

5 Die Analyse der Verfallsformen von Staat und Gesellschaft bei Platon und Aristoteles

5 a. Der Unwille zu unterscheiden als Ursache des Verfalls von Individuum und Staat nach Platon

5 b. ,Der Markt' als Nivellierung des Wertes menschlicher Arbeit nach Aristoteles

Schluß Über eine Grunddifferenz in der Entwicklung des europäischen Denkens: Repräsentations- und Unterscheidungsphilosophien - Eine kurze Rekapitulation der Hauptthese und ihrer Begründung

Literaturverzeichnis 541

I Antike Primärtexte

II Sonstige

Sach- und Personenregister 


\section{Vorwort}

Trotz vieler Bewunderung und Hochschätzung, die Platon immer erfahren hat, ist das Verhältnis, das die Neuzeit und die Moderne gegenüber Platon einnehmen, gebrochen. Die Distanzierung von Platon ist dabei für ein modernes Denken nichts Nebensächliches. Denn es ist geradezu ein Dogma jedes kritischen Denkens, daß es so etwas wie ein für sich seiendes Wesen der Dinge nicht geben könne, und daß es auf jeden Fall nicht erkennbar sein könne. Genau dafür aber steht Platon: er ist der eigentliche Vertreter einer vorkritisch dogmatischen Philosophie, die noch der Meinung anhängen konnte, es gebe allein der Vernunft zugängliche transzendente Substanzen, Wesenheiten, Ideen, deren Kenntnis eine zureichende Erklärung der Welt ohne Rückgriff auf die Empirie - möglich machten. Mit der Entstehung und der Berechtigung dieses Platonbildes, das sich seit dem späten Mittelalter konsequent verfolgen läßt, befaßt sich dieses Buch. Die Darstellung ist aber nicht ausschließlich historisch. Es geht nicht um eine Rezeptionsgeschichte Platons, die in einer einzigen Monographie gar nicht gegeben werden könnte. Im Zentrum steht vielmehr die Sachauseinandersetzung. Welche Gründe haben zu diesem - wohl verzerrten - Platonbild geführt, welches Gewicht kommt den Argumenten zu, die zu diesem Bruch mit dem Platonismus geführt haben, und welche Folgen hat dieser Bruch für das Selbstverständnis, in dem sich das kritische Denken der ,Moderne' gegen Platon abgrenzt? In diesem Sinn will dieses Buch auch parteiisch sein, wenn auch nicht im Sinn einer willkürlichen Parteinahme, wohl aber im Sinn einer Abwägung des besser und des weniger gut Begründeten und eines Plädoyers für das, was aufgrund belegbarer Argumente besser begründet erscheint.

Die Bezugnahme auf Platon ist dabei nicht der Ausgangspunkt der Arbeit an diesem Buch, sondern Ergebnis scheinbar viel breiter angelegter Untersuchungen. Im Rahmen eines seit vielen Jahren zusammen mit Kollegen und Schülern betriebenen Forschungsprojekts haben wir von verschiedenen Seiten her die Frage zu klären versucht, warum es seit dem Bruch mit dem Mittelalter so viele Äußerungen in wirkmächtigen Texten gibt, die die aus diesem Bruch entstandene ,Moderne' in einer ,querelle', einer direkten Auseinandersetzung mit der Antike sehen. Allein der Überblick über die Verbreitung der verschiedenen Formen dieser, querelle' machte deutlich, daß es sich hier um ein für das Selbstverständnis der Moderne wesentliches Bewußtsein handelt: Es ist nicht nur die Antike, gegen die sich die nachmittalterliche ,Moderne' in einem scharfen Gegensatzbewußtsein abgrenzt, diese Abgrenzungsgewohnheit der Moderne erstreckt sich vielmehr seither auf beinahe alles Nichtmoderne, und so auch auf alle Kulturen, die nicht aus der europäischen, westlichen Kultur hervorgegangen sind. Dabei spielt es meistens keine Rolle, ob die Antithese als Überwindung des Alten durch die Moderne gerühmt oder nostalgisch als Verlust ursprünglicher Lebensfor- 
men beklagt wird, maßgeblich bleibt für die positive wie die negative Wertung das gleiche direkte Oppositionsbewußtsein, das sich von ,der Antike' oder anderen nichtmodernen Kulturen in ihrer Gesamtheit abgrenzt.

Auch geschichtlich beschränkt sich das Bewußtsein des Traditionsbruchs zusammen mit den (Vor-)Urteilen, in denen der Zustand vor und nach diesem Bruch formuliert wird (, aufgeklärt-unmündig', , naiv-reflektiert', ,konventionell-individuell' ${ }^{\prime}$ usw.), nicht auf eine einmalige Epochengrenze zwischen ,der' Antike und ,der' Moderne, sondern es beherrscht (seit dem 18. Jahrhundert in zunehmender Beschleunigung) auch das Verhalten jeder einzelnen Epoche innerhalb der Moderne zu ihrer eigenen unmittelbaren Vergangenheit und damit zu jeder Form von Vergangenheit.

Wer sich auch nur mit irgendeiner Debatte beschäftigt, die in gegenwärtigen ,Diskursen' in der Philosophie, den Künsten, in den Naturwissenschaften, der Politik, Ökonomie usw. geführt wird, wird unweigerlich mit zwei Befunden konfrontiert. Der erste ist, daß es eine gemeinsame Grunddiagnose für alle Problembereiche gibt. Diese Diagnose lautet: Wir leben in einer Phase des Umbruchs, der Krise, der permanent sich beschleunigenden Veränderung. Der zweite Befund ist, daß der in der Gegenwart erreichte neueste Zustand - einer Wissenschaft, einer künstlerischen oder politischen 'Bewegung' usw. - als Resultat einer ,Wende', eines ,Paradigmenwechsels', einer ,Revolution' dargestellt wird: Die neueste Gehirnforschung bietet nicht nur eine vielleicht markante Verbesserung dessen, was ihre unmittelbare Vorgängerin erreicht hatte, sondern sie macht ,Entdeckungen', die die alte Position, revolutionär' verändern und ein völlig neues ,Paradigma' einführen. Analoges gilt in beinahe jedem Bereich.

Neu ist dieses Denken, das Veränderung vor allem als Revolution begreift, freilich keineswegs. Nicht nur das 20. Jahrhundert bietet eine ganze Fülle solcher ,Wenden': die Wende zum Unbewußten (Freud), die Wende zur Sprache (Wittgenstein), die leibphilosophische Wende (von Herder bis Gernot Böhme), die Wende zum Gefühl (emotionale Intelligenz') und neuerdings die Wende zum Bild (Gottfried Böhm, Hans Belting u.a.), auch Darwins besondere Leistung kann nach der Ansicht der meisten nur in Begriffen einer revolutionären Wende - von einer statisch dogmatischen zu einer evolutionär entwicklungsgeschichtlichen Erklärung des Lebens - beschrieben werden. Denselben Anspruch hat schon Kant für sich selbst erhoben und dabei das Vorbild aller dieser Wenden benannt, nach dem Hans Blumenberg die ganze Moderne charakterisiert hat: die kopernikanische Wende. In der Tat läßt sich ein solches Revolutionsbewußtsein an fast allen wichtigen Veränderungvorgängen seit der frühen Neuzeit kontinuierlich bis in die jüngste Gegenwart belegen.

Was ist der Grund, warum - anders als in fast allen anderen Geschichtszeiträumen - in dieser knapp 700 Jahre alten Phase der Neuzeit und Moderne so häufig das Bewußtsein anzutreffen ist, das Neue, ,Moderne' könne nur durch eine entschiedene Abkehr vom Alten, durch seine Destruktion oder Überwindung erreicht werden? Dieser Frage stellt sich das For- 
schungsprojekt, dessen Hauptresultat in diesem Buch zur Diskussion gestellt werden soll.

Die Grundfrage ist, warum in vielen Texten vom 14. bis ins 20. Jahrhundert, in denen der Anspruch erhoben wird, ein ,modernes' Entwicklungsniveau zu vertreten, dieser Anspruch mit dem Bewußtsein verbunden ist, das nichtmoderne Vergangene in einer revolutionären Wende außer Kraft gesetzt zu haben, mit der Folge, daß das Bewußtsein der Modernität fast immer mit dem Bewußtsein eines Traditionsbruchs verbunden ist: Das Vergangene scheint in einer Weise überholt, die eine direkte Sachauseinandersetzung nicht mehr möglich macht. Man kann im Vergangenen viel Interessantes und Nacherlebenswertes finden, verbindliche Relevanz für die Lösung gegenwärtiger Probleme scheint ihm nicht mehr zukommen zu können.

Eine kritische Auseinandersetzung mit diesem Geschichtsverlust in der Moderne, die nicht auf nostalgische Klagen beschränkt ist, ist bisher kaum in Angriff genommen. Verfolgt man die geschichtlich belegbaren Vorgänge, die zu dieser Art von Modernitätsbewußtsein geführt haben, und prüft die jeweils vorgebrachten Sachgründe, die zur vermeintlich endgültigen Überwindung des Alten geführt haben, kann man die Feststellung machen, daß es sich bei diesen Vorgängen keineswegs um geschichtlich notwendige Entwicklungen - auf einen Stand, hinter den ein modernes Denken nicht mehr zurückfallen darf - handelt, sondern um zum Teil hochproblematische Prozesse, die die Moderne mit einer Vielzahl von Hypotheken belastet haben, die für beinahe die meisten Aporien noch der Gegenwart verantwortlich sind. Als der zentrale Umbruch, aus dem fast alle weiteren Wenden der Moderne Ableitungen sind bzw. den sie variieren, erweist sich die frühneuzeitliche ,Wende des Denkens auf sich selbst', d.h., die Überzeugung, in einem revolutionären Bruch mit dem Mittelalter (und dessen Abhängigkeit von der Antike) überhaupt erst die Möglichkeit einer souveränen Selbstbestimmung des Menschen begründet zu haben.

Der zentrale Impuls in dieser Wende auf sich selbst ist die ausschließliche Anerkennung der empirischen Wirklichkeit und der eigenen, individuellen Existenz im Hier und Jetzt als Maßstab des Erkennens und Handelns: Nur das, was gegenwärtig und sinnlich erfahr- und nachprüfbar existent ist, wird als Wirklichkeit und als etwas, woraus das Denken seine Begriffe gewinnen kann, anerkannt. Diese Überzeugung war ursprünglich und ist bis heute der Intention nach ein Anti-Platonismus: sie bestreitet die platonische Lehre von der Wirklichkeit der Ideen, d.h., einer ,Sache selbst', die auf rein rationalem Weg erkennbar ist, und besteht in Opposition zu dieser auf der unmittelbaren Evidenz der sinnlich erfahrbaren Einzeldinge. Historisch betrachtet hatte die Entwicklung, in der sich dieser Anti-Platonismus zur beinahe einhelligen Überzeugung durchsetzte, mehrere Phasen. In diesen aber blieb und bleibt, auch nachdem man glaubte, diese spekulativen Theorien endgültig überwunden zu haben, Platon immer der Antipode, in Auseinandersetzung mit dem jeweils die eigene ,kritische Wende' vollzogen wird. 
- Es läßt sich zeigen, und ich möchte dies auch an einer Reihe von Fällen vorführen, daß es in der Neuzeit eine Folge von immer wieder neu vollzogenen Wenden gibt, die eine gleiche Grundfigur wiederholen.

Wenn also in vielen Texten die ,Moderne' dadurch charakterisiert und von anderen Epochen abgegrenzt wird, daß sie als eine Zeit der kritischen Wende des Denkens auf sich selbst und des radikalen Bruchs mit der Vergangenheit beschrieben wird, dann ist dabei immer eine direkte Gegnerschaft zu Platon mitgemeint, auch wenn dies nicht immer thematisiert wird. Dies gilt besonders für den mittelalterlichen Universalienstreit, der der unmittelbare Anlaß für den Bruch mit dem Mittelalter und den ,Aufbruch' in die frühe Neuzeit war.

Charakterisiert ist die nominalistische Wende, mit der der unter den Aristotelikern des 13. und 14. Jahrhunderts geführte Streit um eine korrekte Aristotelesauslegung ,beigelegt' wurde, durch die ,endgültige' Destruktion jeder Art von Universalienrealismus. ,Universalienrealismus' meint, daß Universalien, d.h. Allgemeinbegriffe, eine vom menschlichen Denken unabhängige, ,reale' Seinsweise haben sollen. Dem setzen das späte Mittelalter und die frühe Neuzeit die These entgegen, daß Begriffe immer nur subjektive Produkte sind, die das menschliche Denken selbst erst aus der Abstraktion von Einzeldingen gebildet hat. Als Begründer der Lehre, daß Begriffe ein eigenes ideales Sein haben, gilt Platon. Das heißt: es setzt sich in dieser ,scholastischen' Debatte eine Position durch, die der Intention nach antiplatonisch ist.

Eine, weichere' Form dieses Universalienrealismus sollen Aristoteles und die ihm folgende mittelalterliche Scholastik ausgebildet haben. Sie behaupten vorgeblich nicht, daß Begriffe ein ideales, transzendentes Sein haben, sondern schreiben den, platonischen' Ideen eine Existenz in den Einzeldingen selbst zu: die Idee des Menschen hat ihren Ort nicht in einem geistigen Jenseits, sondern in den vielen existierenden Menschen selbst.

Gegen beide Formen des Universalienrealismus - gegen die Annahme einer transzendenten, ,idealen' Seinsweise des Allgemeinen und gegen die Annahme einer immanenten Realität von allgemeinen Begriffen in den Einzeldingen - wendet sich der Nominalismus mit der These, daß Begriffe Realität nur im Denken haben.

Das geschichtliche Resultat des spätmittelalterlichen Universalienstreits, d.h. die Art und Weise, wie hier Platon ein für alle Mal überwunden worden sein soll, ist bis heute nicht in Frage gestellt worden. Im Gegenteil: wenn es in der pluralen Offenheit der Moderne eine Überzeugung gibt, die niemand mehr kritisiert, zumindest niemand, der sich auf der Höhe seiner Zeit bewegen möchte, dann ist es die Überzeugung, daß Begriffe subjektive Produkte ohne ein eigenes reales Sein für sich selbst sind. Unter diesem Gesichtspunkt kann die Moderne insgesamt seit ihren frühesten Anfängen im 14. Jahrhundert als eine anti-platonische Zeit bezeichnet werden.

Die wirkungsgeschichtliche Kraft dieses Anti-Platonismus ist so groß, daß sie selbst die besonnensten und sorgfältigsten Interpreten davon abhält, 
die ungeheure Masse an historisch zuverlässig belegtem Material zur Kenntnis zu nehmen, durch welches die gesamte Universaliendiskussion, die für den Bruch mit dem Mittelalter verantwortlich ist, als eine grandiose Fehlkonstruktion erwiesen wird. Im Sinn einer ,Bilanz', einer Abwägung des Positiven und Negativen, möchte ich im folgenden einige Ergebnisse langjähriger Forschungen über die Entstehungsbedingungen dieser ,Konstruktion der Moderne' vorlegen und dabei nicht nur zu zeigen versuchen, wie diese Konstruktion zustande gekommen ist, sondern auch, was durch sie aus dem Blick geraten ist und deshalb eine klare Sicht sowohl auf uns selbst als auch auf die vermeintlich überwundene Vergangenheit behindert.

Das beeindruckende Ergebnis dieser ,Archäologie' ist, daß die neuzeitliche Wende zum Einzelding und zum Einzelnen erheblich mehr an spekulativer Metaphysik mit sich führt, als sie sich bei Platon und im Platonismus belegen läßt. Der zentrale Impuls Platons ist, das scheint mir klar und umfassend belegbar zu sein, eine auf das Widerspruchsaxiom gestützte rationale Erklärung gerade des empirisch Einzelnen. In der Neuzeit tritt an die Stelle des rationalen Kriteriums des Widerspruchsaxioms das Einzelding selbst. Es gibt die Einheit vor, der die Begriffe des Denkens gemäß sein müssen. Von diesem unterschiedlichen Ausgangspunkt her ergeben sich zwei völlig verschiedene Auffassungen von Rationalität und, diesen folgend, von den anderen Vermögen des Menschen: Wahrnehmung, Gefühl, Wille usw. Diese unterschiedlichen Formen von Rationalität - die man als eine abstrakte und eine konkrete Rationalität charakterisieren kann - mit ihren Folgen für das unterschiedliche Selbstverständnis des Menschen herauszuarbeiten, ist das Hauptanliegen dieses Buchs. (Um den Grundansatz, aus dem heraus diese beiden unterschiedlichen Formen von Rationalität jeweils hervorgegangen sind, auch mit einem charakteristischen Begriff $\mathrm{zu}$ verbinden, schlage ich für die eine Form den Begriff ,Repräsentations- oder Vorstellungsphilosophien', für die andere den Begriff ,Unterscheidungsphilosophien' vor.)

Man kann sich dieser Aufgabe nicht stellen, ohne langfristige Traditionslinien und vielfältige interdisziplinäre Bezüge zu berücksichtigen. Gerade weil es um grundlegende, gemeinsame Fragestellungen geht, mußten philosophische, ästhetische, ethische, politische, ökonomische und naturwissenschaftliche Aspekte einbezogen werden. Um die Darstellung auch interdisziplinär verständlich zu halten, habe ich versucht, die Sachprobleme so darzustellen, daß sie aus ihnen selbst heraus verfolgt werden können, ohne daß eine Einarbeitung in die verschiedenen Wissenschaftstraditionen nötig ist. Auch auf eine umfassende Auseinandersetzung mit der wissenschaftlichen Literatur habe ich aus diesem Grund verzichtet. Immer dort, wo es für die Kontrolle der These nötig ist und ich mich nicht auf wohlbekannte, sondern auf entlegenere Positionen beziehe, habe ich aber die erforderlichen Belege zu geben versucht. Ausführlicher dokumentiert habe ich die Forschungsliteratur, wo ich Ergebnisse aktueller Diskussionen aufgreife. Den Versuch, die manchmal differenzierte Struktur der Sachprobleme selbst zu vereinfachen, 
um sie leichter zugänglich zu machen, habe ich aber, jedenfalls mit Absicht, nicht unternommen.

$\mathrm{Zu}$ vielen Ergebnissen, die ich hier vortrage, habe ich wichtige Anregungen von den engagierten Mitarbeitern an dem Projekt ,Neuzeitliches Selbstverständnis und Deutung der Antike', aus ihren Beiträgen in Seminaren und Übungen und ihren Bearbeitungen vieler Themen in Dissertationen, Habilitationsschriften, Monographien und wissenschaftlichen Aufsätzen bekommen, für die ich mich herzlich bedanken möchte. Danken möchte ich auch Reinhard Brandt, Wolfgang Bernard und Friedrich Uehlein für eine kritische Lektüre und ganz besonders Gyburg Radke, die nicht nur in formaler Hin. sicht den Text in Ordnung gebracht und die Indices erstellt hat, sondern vor allem durch substantielle, kritische Ergänzungen und Korrekturen geholfen hat, die Schlüssigkeit der Argumente zu verbessern und Fehler zu vermeiden.

Außerdem danke ich der Deutschen Forschungsgemeinschaft, die mir ein Forschungssemester gewährt hat, durch das mir die Fertigstellung dieses Buches ermöglicht wurde.

1 Die bisher im Rahmen dieses Projekts erschienenen Arbeiten (Bücher und Aufsätze) sind im Literaturverzeichnis mit einem " $"$ " markiert. 